\title{
LA CIENCIA DE LOS ALIMENTOS GEORREFERENCIADA. APROXIMACIÓN BIBLIOMÉTRICA A NIVEL INSTITUCIONAL
}

\author{
The food science georeferenced. A bibliometric \\ approach at institutional level
}

Vicente P. Guerrero-Bote, Carlos Olmeda-Gómez y Félix De-Moya-Anegón

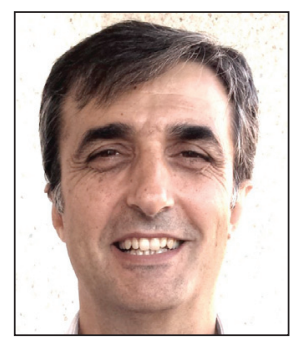

Vicente P. Guerrero-Bote es doctor en documentación por la Universidad de Granada, y licenciado en física por la Universidad Complutense de Madrid. Es catedrático de evaluación de la investigación y análisis de redes de la Universidad de Extremadura, donde es decano de la Facultad de Ciencias de la Documentación y la Comunicación. Es miembro del SCImago Research Group, dedicado a cienciometría, siendo uno de los desarrolladores del SCImago Journal Rank. http://orcid.org/0000-0003-4821-9768

Univ. de Extremadura, Facultad de Ciencias de la Documentación y la Comunicación Depto. de Información y Comunicación Grupo SCImago

PI. Ibn Marwan, s/n. 06001 Badajoz, España guerrero@unex.es

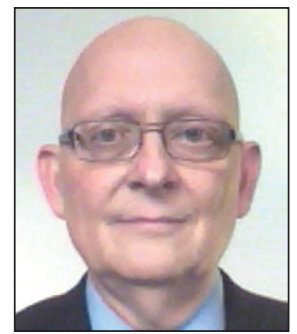

Carlos Olmeda-Gómez es profesor titular en el Departamento de Biblioteconomía y Documentación, en la Facultad de Humanidades, Comunicación y Documentación de la Universidad Carlos III de Madrid. Sus intereses de investigación se centran en los estudios sobre comunicación académica, bibliometría y visualización de información. http://orcid.org/0000-0001-5955-6423

Univ. Carlos III de Madrid, Facultad de Humanidades, Comunicación y Documentación Depto. de Biblioteconomía y Documentación. C/ Madrid, 128. 28903 Getafe (Madrid), España olmeda@bib.uc3m.es

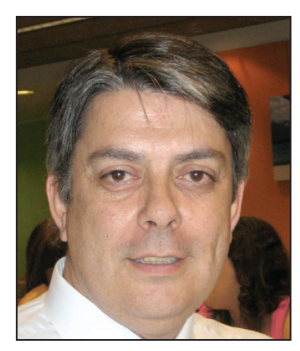

Félix De-Moya-Anegón, doctor en historia por la Universidad de Granada, es profesor de investigación en el Instituto de Políticas y Bienes Públicos del Consejo Superior de Investigaciones Científicas (CSIC) en Madrid, e investigador principal de la unidad asociada SCImago Research Group. Ha dirigido varios proyectos de I+D+i, entre ellos SCImago Journal \& Country Rank (SJR), SCImago Institutions Rankings (SIR), Atlas de la Ciencia, etc.

http://orcid.org/0000-0002-0255-8628

$$
\begin{array}{r}
\text { Centro de Ciencias Humanas y Sociales (CCHS), CSIC } \\
\text { SCImago Research Group } \\
\text { Albasanz, 26-28. 28037 Madrid, España } \\
\text { felix.moya@scimago.es }
\end{array}
$$

\section{Resumen}

Se presenta un análisis bibliométrico de la producción en revistas científicas de la categoría temática food science (ciencia de los alimentos) de Scopus entre 2003 y 2013 a partir de setenta y una instituciones españolas con al menos cincuenta trabajos publicados. Se han empleado pruebas estadísticas z para comprobar el grado en que los trabajos altamente citados de las instituciones, difieren de los valores esperados sobre la base de la aleatoriedad en la selección de artículos. Se ha generado un mapa con un cierto grado de interactividad, en el que se visualizan las redes de colaboración interinstitucional, así como los valores de actividad e impacto. Los resultados muestran los centros más activos, ubicados principalmente en Madrid, Cataluña, Valencia y Murcia, pertenecientes al CSIC y a los centros de investigación de Cataluña.

\section{Palabras clave}

Ciencia de los alimentos; Análisis de citas; Bibliometría; Redes de colaboración; Excelencia científica; Mapas bibliométricos; Geo-referencia. 


\begin{abstract}
A bibliometric analysis of research production in scientific journals, of the Scopus subject category Food Science, between 2003 and 2013, from seventy-one Spanish institutions with at least fifty works published, is presented. Statistical z tests are used for an evaluation of the degree to which an observed number of top-cited papers (top-10\%) for an institution differs from the number expected, on the basis of randomness in the selection of papers. A map has been generated that displays inter-institutional collaboration networks; activity and impact values are displayed. The results show the most active institutions, located primarily in Madrid, Cataluña, Valencia, and Murcia, belong to the Spanish National Research Council (CSIC) and Catalonian research institutions.
\end{abstract}

\title{
Keywords
}

Food science; Citation analysis; Bibliometrics; Coauthorship networks; Research excellence; Bibliometrics maps; Geo-code.

Guerrero-Bote, Vicente P.; Olmeda-Gómez, Carlos; De-Moya-Anegón, Félix (2016). "La ciencia de los alimentos georreferenciada. Aproximación bibliométrica a nivel institucional”. El profesional de la información, v. 25, n. 1, pp. 25-34.

http://dx.doi.org/10.3145/epi.2016.ene.04

\section{Introducción}

En España, la industria de la alimentación es el primer sector industrial, una de las principales fuentes económicas de prosperidad nacional y un sector estratégico. Proporciona empleo a 445.475 personas, con 30.261 empresas (siendo pymes el $96 \%$ ) y es el segundo sector español exportador, por detrás de la automoción (Muñoz-Cidad; Sosvilla-Rivero, 2012). En consecuencia, la investigación y el desarrollo en este sector y en sectores afines, tienen una elevada prioridad en las políticas de ciencia y tecnología de España, siendo ejemplo de ello los proyectos de investigación financiados en el sector con programas nacionales (Cenit, Consolider, Innpronta) y europeos.

En la bibliografía no se encuentran muchos estudios bibliométricos sobre ciencia de la alimentación a nivel institucional. Los más comunes son los que analizan de forma comparativa las producciones científicas a nivel nacional. Por ejemplo Alfaraz y Calviño (2004) realizan un estudio sobre países iberoamericanos, entre 1991 y 2000, con datos de la base de datos Food Science and Technology Abstracts. Concluyen que España tiene más de la mitad de los registros y que en esa década tiene una tasa de crecimiento del $11 \%$ anual. En la Unión Europea, a partir del proyecto Agrimapping en 2006, se recabó información estadística sobre las actividades del sector, con especial interés en los países que se habían incorporado a la Unión de la Europa Central y del Báltico (Borsi; Schubert, 2011).

Con datos procedentes de la Web of Science, se ha analizado la producción mundial en la categoría Agronomía, entre 1997 y 2011 (Cañas-Guerrero et al., 2013). Según el estudio, el Consejo Superior de Investigaciones Científicas (CSIC), aparece entre los diez centros mundiales con mayor actividad de producción en la categoría. Sobre la producción y colaboración científica en Agroalimentación en España, se realizó un trabajo con datos de la Web of Science entre 1990-2005, basado solamente en el impacto esperado para medir el rendimiento de la investigación (Chinchilla-Rodríguez; Olmeda-Gómez, 2010).

Este trabajo persigue analizar la producción científica de centros de investigación en España en la categoría temática Food Science de Scopus. De este modo conocemos el grado de reconocimiento internacional que tiene su producción científica. En concreto, nos preguntamos:

1. ¿Qué posición ocupan los diferentes centros españoles que tienen actividad de publicación en revistas científicas de ciencia de los alimentos?

2. ¿Cuál es el rendimiento de la investigación de los centros españoles? ¿Existen diferencias?

3. ¿Qué impacto logran las colaboraciones que se establecen a partir de realizar trabajos con coautorías? ¿Con qué países extranjeros se colabora más y qué impacto se logra en virtud de esa colaboración?

Para responder estas preguntas, se presenta primero la metodología y luego se discuten los resultados más significativos.

\section{SClmago Institutions Rankings incluye indicadores bibliométricos de 4.289 cen- tros de investigación de todo el mundo, incluidas universidades e institutos}

\section{Métodos y materiales}

El material empírico que se usa en este trabajo se basa en datos originales de la base de datos multidisciplinar Scopus, compilados para el SCImago Institutions Rankings (SIR). http://www.elsevier.com /solutions/scopus http://www.scimagoir.com

Scopus es la base de datos de resúmenes y de citas más amplia por cobertura de títulos de revistas académicas revisadas con peer review y es publicada por Elsevier. SCImago Institutions Rankings incluye indicadores bibliométricos de 4.289 centros de investigación, incluidas universidades e institutos dedicados a la investigación de todo el mundo (agosto 2015).

La clasificación temática del SIR sigue las convenciones de Scopus a la hora de clasificar sus revistas en 27 grandes áreas temáticas (Subject Area) y en 313 categorías temáticas más restringidas o menores (Specific Subject Areas or Categories). Dentro del área temática correspondiente a 
Agricultura y Biología (Agricultural and Biological Sciences), que se compone de once categorías temáticas específicas, se encuentra ciencia de los alimentos (Food Science), que en 2013 cuenta con 234 títulos de revistas.

Para los fines descritos, se han descargado todos los documentos publicados en las revistas de la categoría Food Science de Scopus publicados entre 2003-2013 en los que apareciera España en el campo correspondiente de las direcciones de afiliación de los autores. El análisis se realiza sobre un conjunto de 13.077 documentos, de los que el 92\% son artículos, un $2 \%$ son comunicaciones a congresos, un $4 \%$ revisiones y un $1 \%$ son de otros tipo, sin ser editoriales. El $97 \%$ de los trabajos se han publicado en inglés y el $3 \%$ en español.

El modo elegido para delimitar el dominio temático, es objetivo y sigue las convenciones habituales en trabajos bibliométricos, a pesar de que al basarse en la clasificación de revistas pueda conllevar algunos errores por inclusión o exclusión. De modo que no se prejuzgan los resultados alternativos que se pudieran obtener mediante la delimitación del dominio temático por otros procedimientos (YegrosYegros, 2011) o, por ejemplo, el empleo de otras bases de datos.

La producción en ciencia de los alimentos representa un $2 \%$ del total de la producción científica de España

De las instituciones españolas participantes, con el fin de descartar aquellas que tienen una producción en ciencia de los alimentos ocasional, se han tenido en cuenta las que han producido 50 o más trabajos en el período de estudio. De este modo nos quedamos con aquellas que se dedican con regularidad a la misma. En este caso se ha contabilizado un total de 71 instituciones españolas. El 56\% están adscritas al sector de instituciones de educación superior en SCImago Institutions Rankings, el $47 \%$ a institutos públicos de investigación, incluidos 16 institutos subordinados a entidades de investigación de carácter superior y el 0,2\% a instituciones sanitarias.

Los indicadores bibliométricos calculados para caracterizar la producción científica en ciencia de los alimentos de cada una de las instituciones son los siguientes:

- Ndoc: número de documentos publicados en revistas científicas clasificadas en Food Science recogidas en la base de datos de Scopus.

- \%Ndoc: porcentaje que suponen los documentos de la categoría (en este caso Food Science) con respecto al total de la producción de la institución en cuestión.

- Citas por documentos: promedio de citas por documento. La citación depende en gran medida del tiempo que el documento haya tenido para ser citado; por esta razón no se evalúa la evolución de este indicador.

- \% de documentos citados: al igual que el indicador anterior depende en gran medida del tiempo que los documentos han tenido para ser citados, por esta razón no se evalúa su evolución.
- \% colaboración internacional (IC): porcentaje de documentos en los que en las direcciones institucionales de los autores de los trabajos aparecen autores de varios países. La contabilidad de las ocurrencias se realiza mediante el procedimiento whole counting, siguiendo los procedimientos de cómo ha obtenido y asignado Scopus las direcciones personales contenidas en las publicaciones que contiene su base de datos. Si existen dos instituciones firmantes diferentes en las publicaciones, se usan los dos nombres para añadirles posteriormente las coordenadas geográficas de longitud y de latitud.

- Garante de la investigación (RG): número de documentos publicados en revistas científicas recogidas en Scopus en los que un autor de la correspondiente institución ha actuado como garante de la investigación (autor de la correspondencia) (De-Moya-Anegón et al., 2013). El indicador se expresa también como porcentaje (\% RG).

- Impacto normalizado (NI): promedio de la citación normalizada recibida por cada documento. Entendiéndose ésta como la relación entre la citación recibida por el documento y la citación promedio de los documentos del mismo tipo, año y categoría temática (Rehn et al., 2014).

- Excelencia10: número de documentos que se encuentran entre el $10 \%$ más citado en la misma categoría, mismo año y tipo de documento (Bornmann; De-Moya-Anegón; Leydesdorff, 2012). El indicador se expresa también como porcentaje (\% Exc10).

- Excelencia10 como RG: número de documentos que están entre el $10 \%$ más citado en la misma categoría, mismo año y tipo de documento. El autor de la institución correspondiente, debe haber actuado como garante de la investigación (autor de la correspondencia). El indicador se expresa también como porcentaje (\% Exc10 RG).

- Excelencia1: número de documentos que están entre el $1 \%$ más citado en la misma categoría en el mundo, mismo año, y tipo de documento. El indicador se expresa también como porcentaje (\% Exc1).

- Tasa de variación: para ver la evolución de los indicadores anteriores, salvo las excepciones mencionadas, en este período se ha calculado una tasa de variación. Ésta se ha calculado como la variación de valores entre el promedio del primer trienio (2003-2005) y el último trienio (20102012). Se ha evitado utilizar el año 2013, porque la citación de este año no se puede considerar estable en el momento en el que se descargaron los datos.

Para evaluar el grado en el que el número observado de trabajos Exc10 de una institución difiere del que podría esperarse sobre la base de la aleatoriedad en la selección de trabajos en su cálculo, se ha practicado la prueba $Z$ para dos proporciones independientes. De este modo inferimos qué instituciones españolas tienen un rendimiento sobresaliente respecto de la excelencia científica (Exc10), basándonos en esta prueba estadística (Bornmann; Leydesdorff, 2011).

En estudios de informetría se emplean datos heterogéneos, entrelazados, complejos y voluminosos. Para obtener resultados comprensibles, es necesario hacer análisis que lo faciliten. Con el empleo de técnicas de visualización se facilita el logro de ese fin (Olmeda-Gómez, 2014). En este estudio se emplean dos técnicas: 
Con la primera, se representa al conjunto de instituciones españolas con producción en la categoría basándonos en estimaciones de la similitud que existe entre ellas. Se emplea para ello el escalamiento multidimensional. De forma descriptiva, esta técnica se refiere a un conjunto de procedimientos utilizados en el análisis de datos y reducción de dimensiones. Toma como input estimaciones de la similitud entre grupos de items. El resultado es un mapa en el que se sitúan espacialmente más próximos entre sí, los items más similares y los disímiles más alejados. A partir de su interpretación se pueden inferir las dimensiones existentes en el conjunto de datos que se analizan, confirmar hipótesis previas, o examinar de forma subjetiva la organización del espacio que se obtiene como resultado (Levy-Mangin; Varela-Mallou, 2003).

Para realizar el que aquí se incluye:

- se han normalizado los valores de cada indicador y de sus tasas de variación, por el valor máximo;

- se han reducido al $10 \%$ los valores de los indicadores dependientes del tamaño (Ndoc, IC, Exc10, RG, Exc10 RG, Exc1), para evitar que focalice la clasificación;

- se ha realizado una normalización euclídea de cada vector compuesto por 26 componentes, con el fin de eliminar las diferencias en módulo y clasificar cada institución en función de los valores relativos de cada indicador;

- se ha calculado la distancia euclídea entre cada pareja de instituciones.

El algoritmo de escalado empleado ha sido Alscal proporcionado por SPPS v. 20.

A partir de la información bibliométrica obtenida se ha realizado un segundo mapa. La geolocalización, que emplea los nombres de lugares o de las instituciones que aparecen en las direcciones de los artículos de investigación, permite localizar los lugares donde se crea y desde donde se difunde el conocimiento (Frenken; Hardeman; Hoekman, 2009); en este caso son los centros españoles con producción en ciencia de los alimentos. Los nombres y las ubicaciones de las instituciones que aparecen en la firma del campo dirección de los documentos se han extraído una vez normalizados por procedimientos manuales y semiautomáticos. Para generar el mapa se ha utilizado la aplicación online GPS Visualizer, gratuita y accesible en internet.

http://www.gpsvisualizer.com

Se han dado a la aplicación las coordenadas de longitud y latitud de las instituciones, y se le han facilitado datos para la representación de los nodos, de los vínculos, con lo que se tiene la posibilidad de consultar sobre el mapa los indicadores. En el mapa se ha utilizado como coordenadas de los países colaboradores, el promedio del número de instituciones colaboradoras. El proveedor de las coordenadas es Google. El mapa completo obtenido como resultado, con posibilidades de hacer zoom y seleccionar detalles, es accesible en: http://tinyurl.com/pp7z4hh4,

\section{España produce un $6 \%$ de los resultados} mundiales en ciencia de los alimentos

\section{Resultados}

Según los datos contenidos en Scopus entre 2003 y 2013, de las 71 instituciones consideradas en el estudio, el sector público de investigación en España ha acaparado el 100\% del total de publicaciones a nivel nacional. Los datos generales de España, de producción en términos absolutos y relativos entre 2003 y 2013 , se muestran en la figura 1 . Indican un crecimiento sostenido de la producción en términos absolutos. La tasa de variación de la producción ha sido del $126 \%$, mientras que la de Exc10, ha sido del $115 \%$. La producción en ciencia de los alimentos relativa respecto al total de la producción científica de España, apenas tiene fluctuaciones en el período

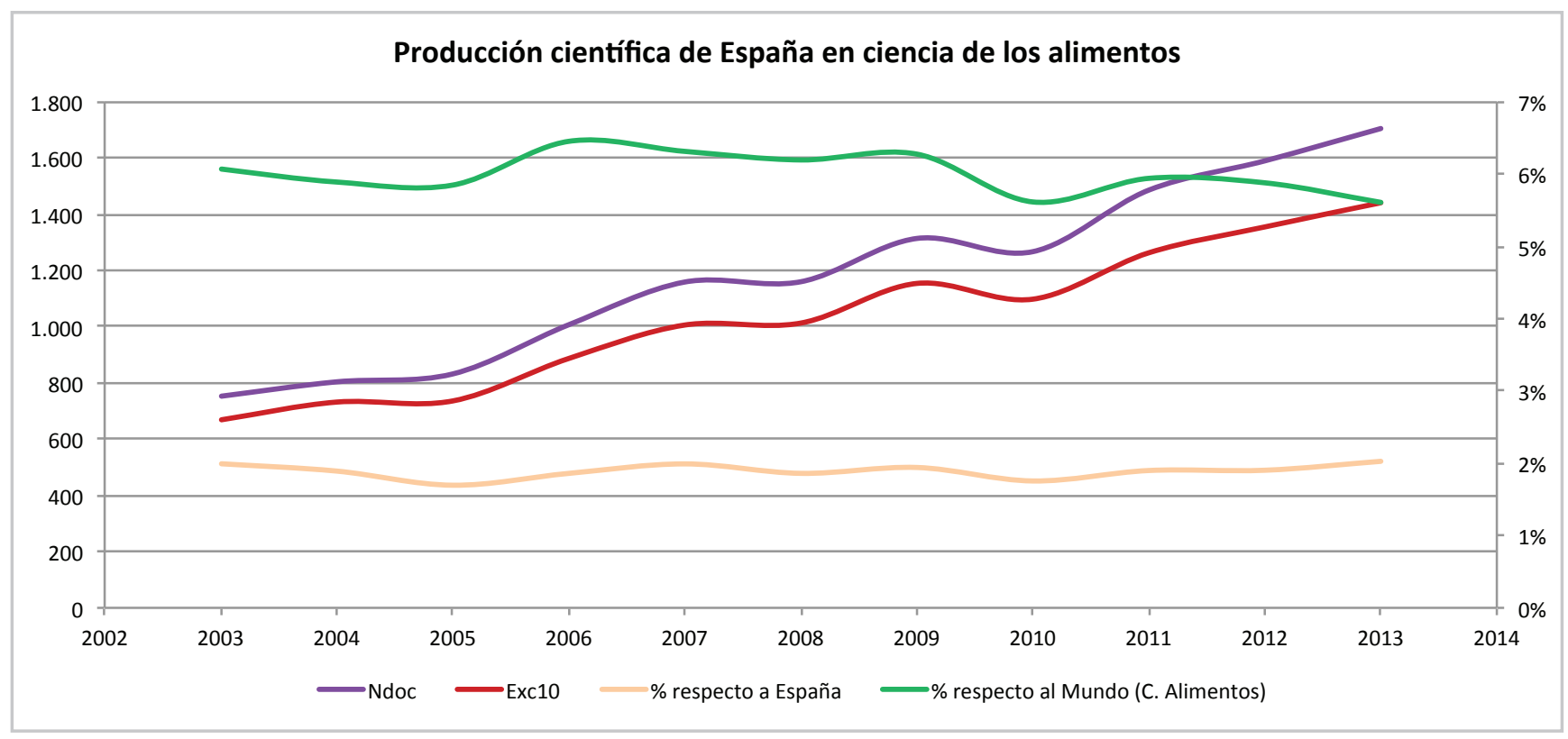

Figura 1. Producción en ciencia de los alimentos en el período 2003-2013. Valores absolutos y porcentuales respecto de España y la producción mundial 


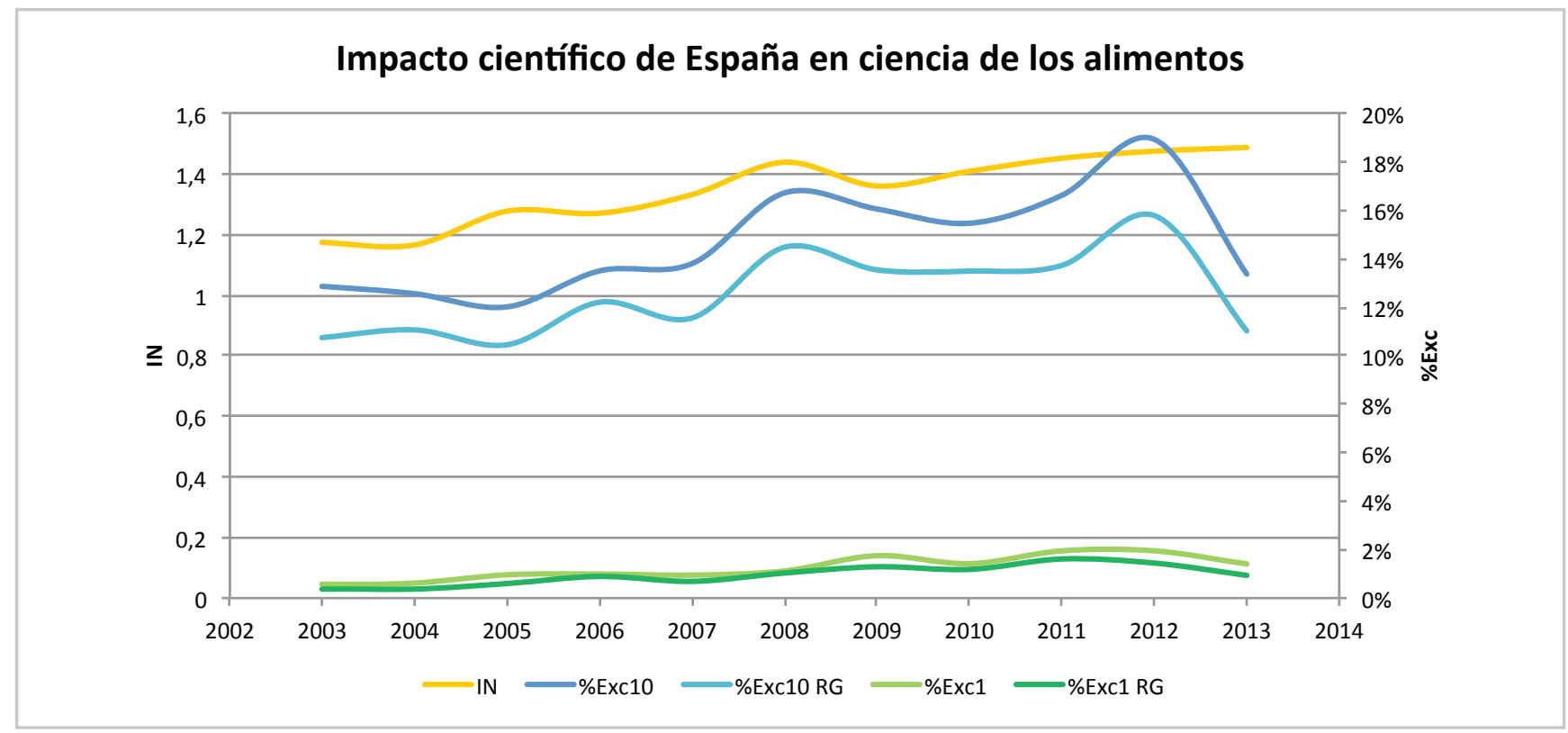

Figura 2. Indicadores de rendimiento de la investigación en ciencia de los alimentos de España en el período 2003-13

y se mantiene estable en torno al $2 \%$. Respecto del mundo, la participación de España disminuye a partir de 2009, como consecuencia del incremento relativo de las producciones de países asiáticos (Malasia, Corea del Sur y sobre todo, China) y de Brasil (Guerrero-Bote; De-Moya-Anegón, 2015). Por sectores, las universidades producen como promedio tres trabajos por institución y los institutos públicos 24 , siendo el promedio nacional de 12 trabajos. En todo caso, el esfuerzo de producción en esta disciplina en España ha sido notable.
Para comprender el impacto según la citación obtenida por la producción en ciencia de los alimentos, se muestra en la figura 2 la evolución de los seis indicadores presentados en la sección de metodología. El valor de impacto normalizado, que es un indicador independiente del tamaño, siempre ha sido superior a lo largo de la década al valor del impacto promedio del mundo, que tendría un valor neutro de 1,0 en todo el período. En 2013 lo supera en 49 puntos, lo que indica que la citación promedio de la producción participada

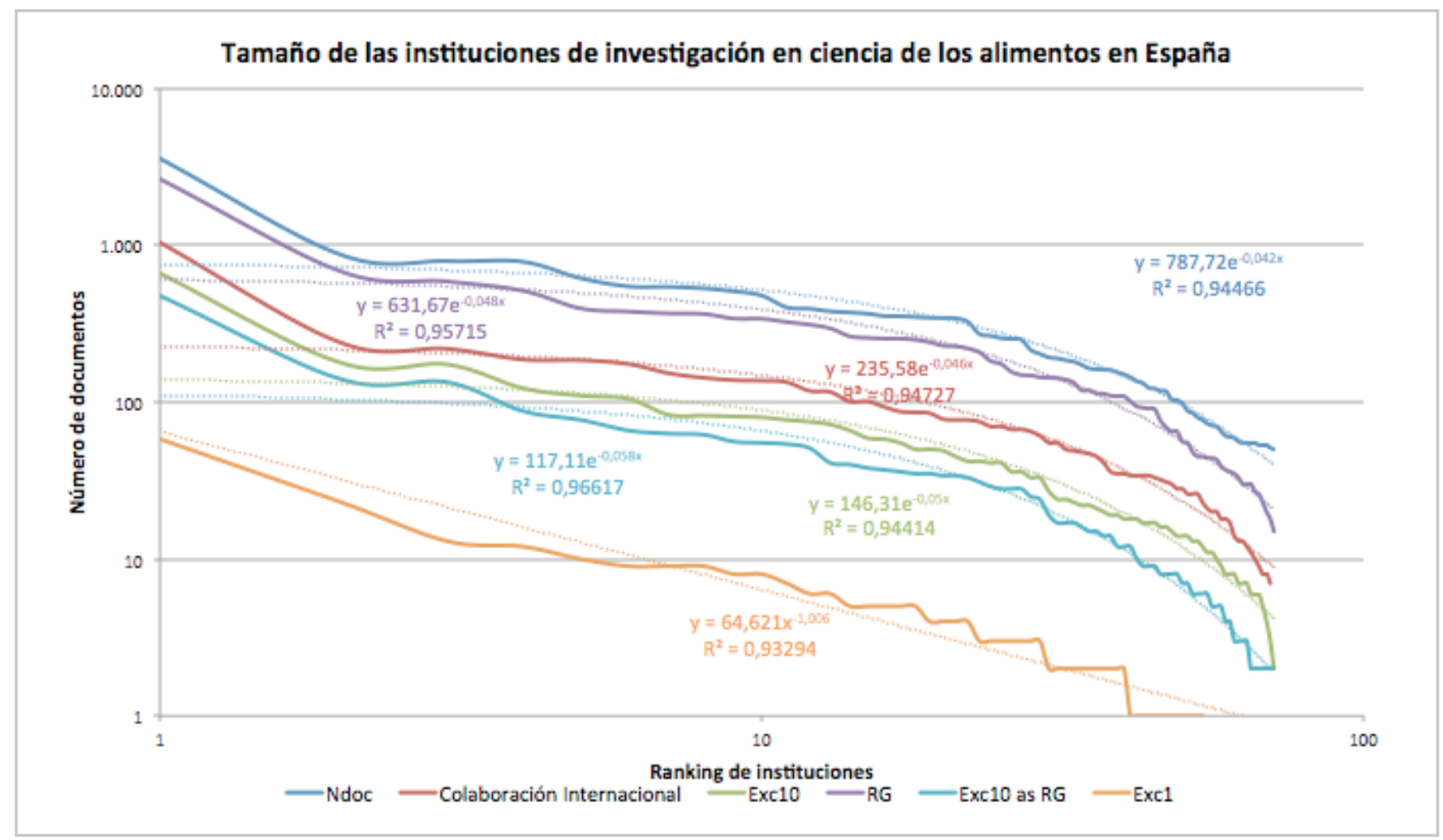

Figura 3. Superposición de los valores de la producción total (Ndoc), producción total como garante de la investigación (RG), producción total con colaboración internacional, producción citable excelente (Exc10), producción citable excelente como garante de la investigación (Exc10 RG), producción citable excelente (Exc1), versus ranking de posiciones de instituciones españolas. Ndoc $>50$ documentos. 


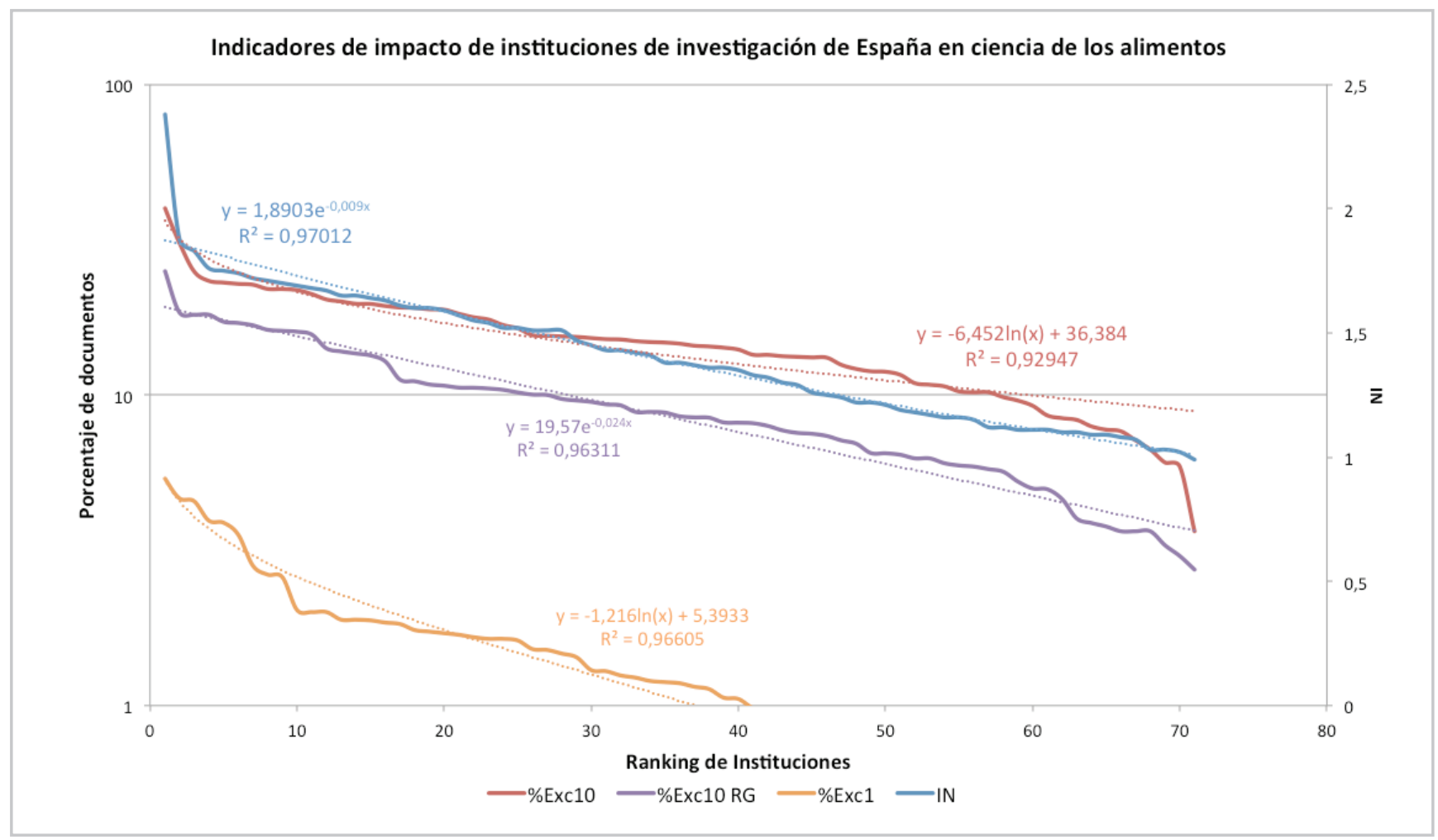

Figura 4. Superposición de valores porcentuales de impacto normalizado (NI), \% de producción citable excelente (\%Exc10), \% de producción citable excelente como garante de la investigación (\% Exc10 RG), \% de producción citable excelente (\% Exc1\%), versus ranking de posiciones de instituciones españolas. $\mathrm{Ndoc}>50$ documentos.

por autores españoles, es casi un $50 \%$ superior al promedio mundial. Su tasa de variación ha sido del $22 \%$. Los volúmenes de Exc10, se han incrementado entre 2003 y 2012 (12,9\% en 2003; 18,9\% en 2012). En el año siguiente, 2013, se produce un descenso acusado, del orden del 5,5\% en los valores del mismo indicador. En menor grado, en 2013 desciende el \%Exc10 RG (15,8 en 2012; 11\% en 2013). También es destacable la evolución positiva del rendimiento medido por el indicador \%Exc1, con una tasa de variación del 153\%, pese a que el mismo sufre un acusado descenso en 2013, respecto del año anterior, a la par del resto de indicadores de excelencia. En gran medida, el descenso en el último año es consecuencia de la poca citación que han recibido todavía los trabajos de 2013. Cuando dispongamos de más años de citación posiblemente se reduzca este descenso. El impacto normalizado promedio a nivel nacional es de 1,40. Los institutos públicos de investigación obtienen 1,47 y la producción participada por instituciones universitarias, el 1,34. Las universidades actúan como garantes de la investigación como promedio en el $69 \%$ de su producción. Los organismos públicos de investigación en un $62 \%$ y la institución sanitaria (Instituto de Salud Carlos III), el 30\%.

Los diagramas de líneas de las figuras 3 y 4 muestran el comportamiento de las 71 instituciones españolas, respecto de indicadores bibliométricos que dependen del tamaño por producción (figura 3) y de indicadores que miden el rendimiento (figura 4). Los indicadores de tamaño tienen una relación positiva cuasi perfecta $(R=\geq 0,93, p<0,01)$, dentro del rango de casos observados. Los indicadores de rendimiento, tienen menores correlaciones entre sí $(R=$ $\geq 0,707, p<0,01$ ).
La zona superior de las distribuciones la ocupan los centros españoles con mayor actividad, presidida por el Consejo Superior de Investigaciones Científicas (CSIC). Son: el Instituto de Ciencia y Tecnología de Alimentos y Nutrición (Ictan), en Madrid, y el Instituto de Agroquímica y Tecnología de Alimentos (IATA), en Paterna, Valencia, ambos dependientes del CSIC. En Cataluña, el centro de referencia es el Instituto de Investigación y Tecnología Agroalimentaria, de Barcelona (IRTA) integrado en la Agencia de Centros de Investigación de Cataluña (Cerca). Se suma a él la Red de Referencia en Tecnologías de los Alimentos, que coordina grupos de investigación existentes en universidades de Cataluña, y la Unidad de Investigación Alimentaria del IRTA. Las universidades también tienen una actividad destacada, como la Politécnica de Valencia, Complutense de Madrid, Lérida, Santiago de Compostela y Zaragoza.

\section{Las universidades con una actividad cien- tífica más destacada en ciencia de los alimentos son la Politécnica de Valencia, Complutense de Madrid, Lleida, Santiago de Compostela y Zaragoza}

Los ajustes de los valores se realizan en un gráfico logarítmico en ambos ejes. En el caso de los valores de Exc1, se ajusta a una línea recta con pendiente acusada, ya que los valores más elevados están muy concentrados, lo que significa que se adapta a una power law. Las caídas de los otros indicadores son más suaves, se adaptan mejor a curvas exponenciales. 
La figura 4 muestra los valores de rendimiento de las principales instituciones con actividad de investigación en ciencia de los alimentos, medidas por cuatro indicadores para calibrar aspectos diferentes del impacto. Es visible que los indicadores de producción con excelencia, se localizan en niveles inferiores en el gráfico a los de la producción absoluta al ser más selectivos. Los indicadores de impacto normalizado NI y de \%Exc10 RG de la parte superior del gráfico muestran un comportamiento cercano a una ley exponencial, que puede representarse en este gráfico semi logarítmico por una línea recta con caída de pendiente pronunciada.

Se revela la importancia que tiene la proximidad cognitiva, organizativa, social, institucional y geográfica, en los procesos de aprendizaje y creación de conocimiento

Existen diferencias altamente significativas $(p<0,001)$ visibles entre los valores observados $\left(v_{o}\right)$ y esperados $\left(v_{e}\right)$ de trabajos de Exc10, en el conjunto de:

- $\operatorname{CSIC}\left(\mathrm{v}_{\mathrm{o}}=654, \mathrm{v}_{\mathrm{e}}=358,2\right)$;

- Centro de Edafología y Biología Aplicada del Segura $\left(v_{\circ}=\right.$ $81, v_{\mathrm{e}}=20,3$ );

- Instituto de Agroquímica y Tecnología de Alimentos (IATA) $\left(v_{\mathrm{o}}=178, \mathrm{v}_{\mathrm{e}}=78,6\right)$;
- Instituto de Ciencia y Tecnología de Alimentos y Nutrición (Ictan) ( $\left.v_{0}=174, v_{e}=87\right)$;

- Universitat de València $\left(\mathrm{v}_{\mathrm{o}}=110, \mathrm{v}_{\mathrm{e}}=47,8\right)$;

- Universitat de Lleida $\left(\mathrm{v}_{\mathrm{o}}=105, \mathrm{v}_{\mathrm{e}}=54,3\right)$;

- Universidad Miguel Hernández $\left(\mathrm{v}_{\mathrm{o}}=75, \mathrm{v}_{\mathrm{e}}=34,29\right)$;

- Red de Referencia en Tecnología de los Alimentos $\left(\mathrm{v}_{\mathrm{o}}=80\right.$, $\left.\mathrm{v}_{\mathrm{e}}=39,4\right)$;

- Universitat de Barcelona $\left(v_{0}=72, v_{e}=36,6\right)$;

- Universitat Rovira i Virgili $\left(\mathrm{v}_{\mathrm{o}}=49, \mathrm{v}_{\mathrm{e}}=21,5\right)$;

- Universidad de Vigo $\left(\mathrm{v}_{\mathrm{o}}=66, \mathrm{v}_{\mathrm{e}}=34,5\right)$;

- Universitat Politècnica de València $\left(v_{0}=123, v_{e}=79,3\right)$.

Diferencias significativas $(p<0,01)$ se encuentran en:

- Instituto de Salud Carlos III $\left(\mathrm{v}_{\mathrm{o}}=22, \mathrm{v}_{\mathrm{e}}=8,8\right)$;

- Instituto de Productos Lácteos de Asturias $\left(\mathrm{v}_{\mathrm{o}}=42, \mathrm{v}_{\mathrm{e}}=\right.$ 19,3);

- Instituto de Investigación y Tecnología Alimentaria de Barcelona $\left(\right.$ IRTA) $\left(\mathrm{v}_{\mathrm{o}}=82, \mathrm{v}_{\mathrm{e}}=53,1\right)$.

Existen también diferencias a favor de trabajos con elevada citación $(p<0,05)$, en:

- Instituto Valenciano de Investigaciones Agrarias $\left(v_{0}=24\right.$, $\left.\mathrm{v}_{\mathrm{e}}=10,3\right)$;

- Universidad de Navarra $\left(\mathrm{v}_{\mathrm{o}}=33, \mathrm{v}_{\mathrm{e}}=17,3\right)$;

- Institut de Recerca i Tecnologia Agroalimentàries, de Monells $\left(\mathrm{v}_{\mathrm{o}}=45, \mathrm{v}_{\mathrm{e}}=25,3\right)$;

- Universidad de Granada $\left(\mathrm{v}_{\mathrm{o}}=58, \mathrm{v}_{\mathrm{e}}=35,4\right)$;

- Universidad de Zaragoza ( $\left.\mathrm{v}_{\mathrm{o}}=77, \mathrm{v}_{\mathrm{e}}=50,9\right)$;

- Universidad de Extremadura $\left(\mathrm{v}_{\mathrm{o}}=55, \mathrm{v}_{\mathrm{e}}=37,3\right)$.

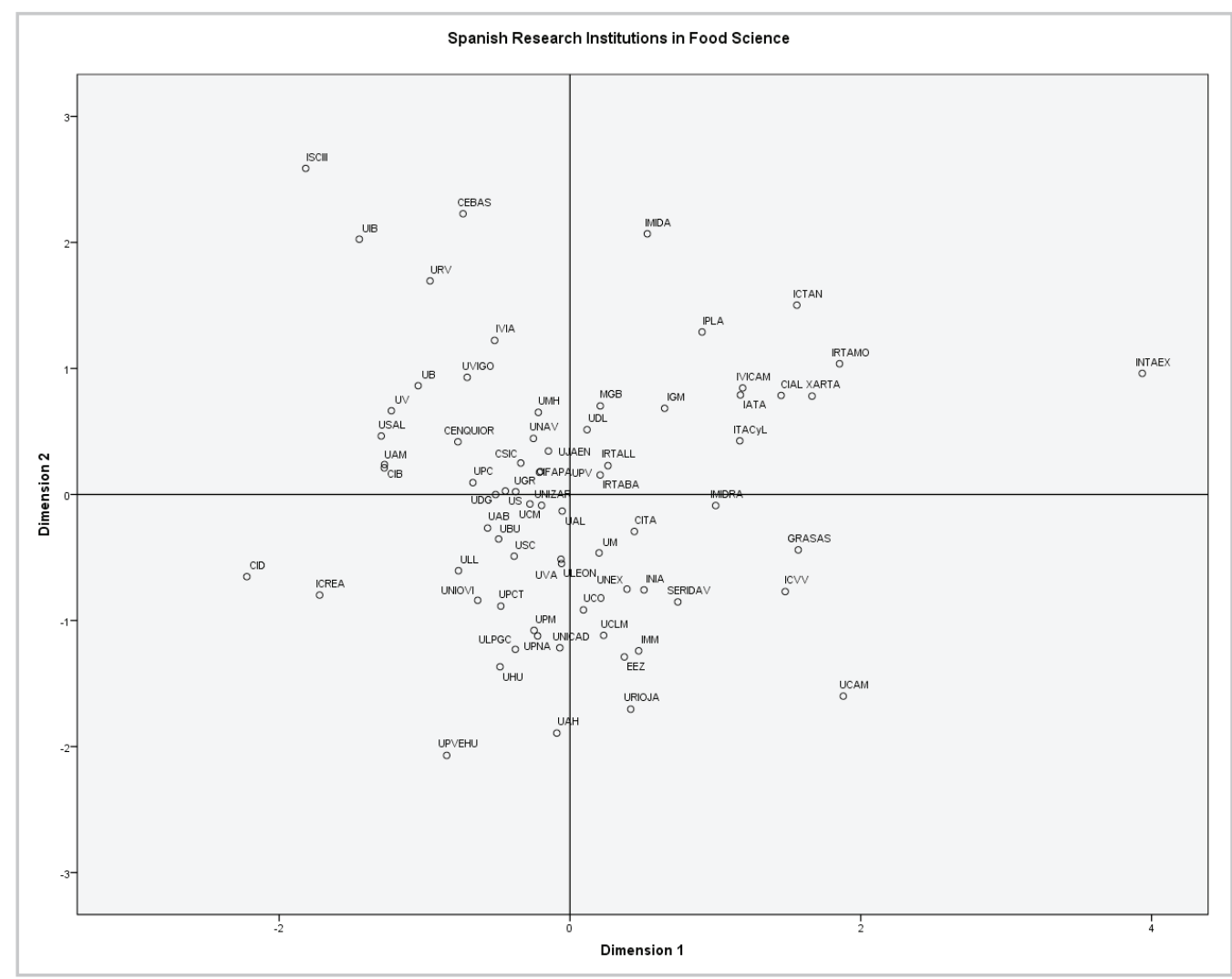

Figura 5. Resultado del multidimensional scaling (MDS) de centros de investigación y universidades españolas utilizando el procedimiento Alscal, y las distancias euclídeas calculadas con valores de indicadores normalizados por el máximo valor y donde se han reducido el peso de los indicadores dependientes del tamaño al 10\% (stress $=0,20968$ ).
En la figura 5 aparece el mapa realizado con la técnica del escalamiento multidimensional (MDS). La primera dimensión parece tener correlación con el grado de especialización de las instituciones, situándose sobre este eje, a la derecha, los centros de investigación, mientras que a la izquierda se sitúan centros universitarios. Las mayores correlaciones de la dimensión 2 son con indicadores de rendimiento, por ejemplo $\mathrm{NI}(0,82)$ y con el resto de indicadores de impacto independientes del tamaño. En la zona superior del mapa se sitúan, pues, instituciones con rendimiento elevado. 


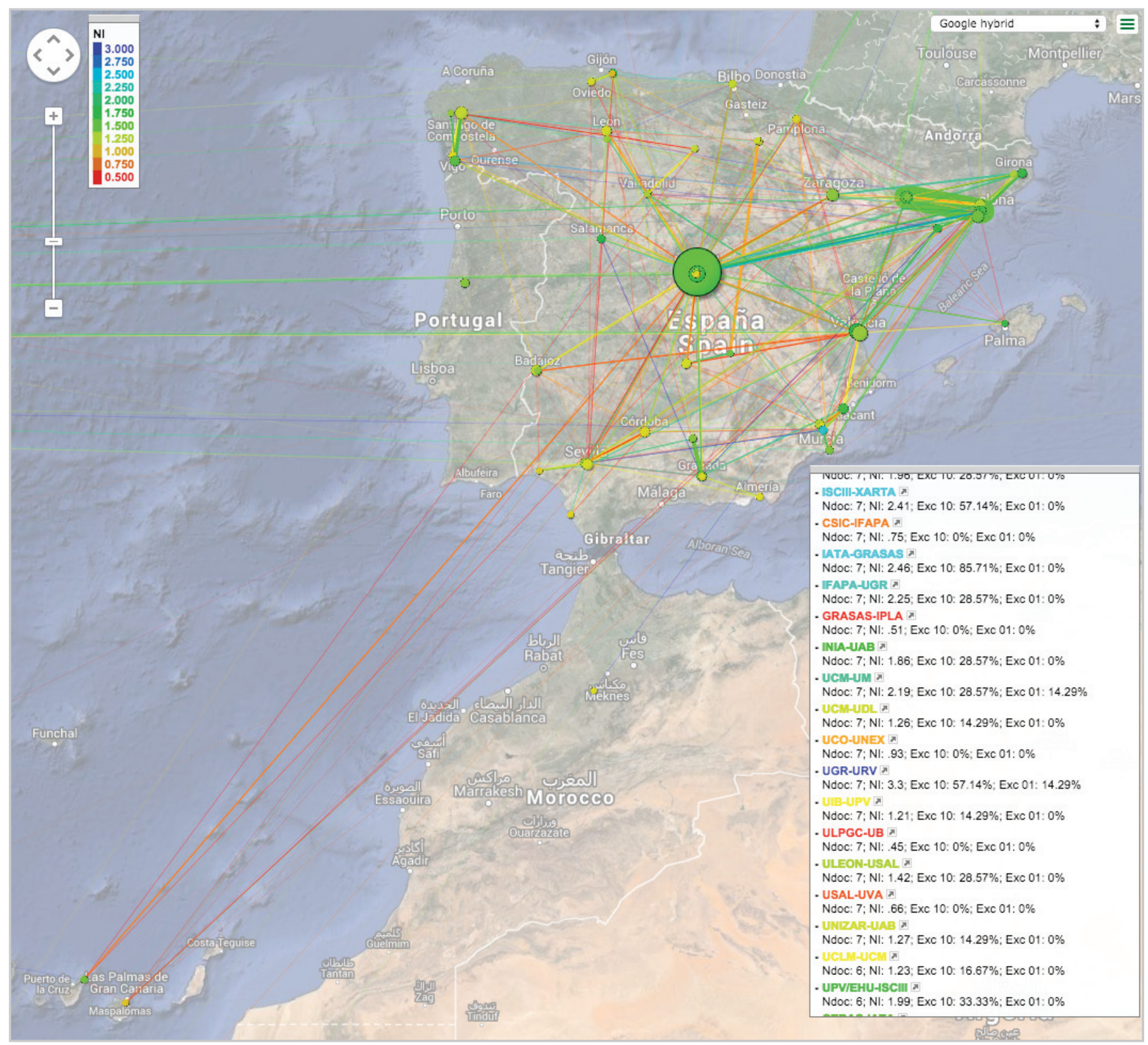

Figura 6. Red española de colaboración interinstitucional de investigación en ciencia de los alimentos 2003-2013 (con una producción total de, al menos, 50 documentos; contabilidades completas). Una versión interactiva, con posibilidades de hacer zoom, es accesible en: $h t t p: / / t i n y u r l . c o m / p p 7 z 4 h h$

El mapa de la figura 6 representa la red española de colaboración interinstitucional de organismos de investigación que han producido, al menos, cien documentos entre 20032013, en publicaciones clasificadas en la categoría temática Food Science de Scopus. En el mapa, el radio de los círculos y el grosor del enlace son proporcionales respectivamente a la producción y volumen de trabajos coautorados entre instituciones. El color del nodo y de los enlaces se corresponde con el valor del impacto normalizado de la producción de la institución respectiva y del valor del impacto normalizado de la producción con colaboración institucional. La equivalencia entre valor del impacto y color aparece situado en la leyenda superior izquierda.

La red nacional se construye desde el punto de vista nodal, fundamentalmente por las producciones del CSIC y de sus institutos, los centros radicados en Cataluña y en la Comunidad Valenciana. El único nodo con valores de $\mathrm{NI}>2,2$, es el que corresponde al centro del CSIC de Edafología y Biología
Aplicada del Segura, en Murcia. Desde el punto de vista de la intensidad en las colaboraciones, destaca la que se establece entre centros radicados en Cataluña con los valores máximos en relaciones de colaboración bilaterales de toda España (por ejemplo, Xarxa de Referència en Tecnologia dels Aliments - Universitat de Lleida, Ndoc: 229; Institut de Recerca i Tecnologia Agroalimentàries, Lleida - Universitat de Lleida: Ndoc: 131). En el resto de España, las relaciones bilaterales entre los investigadores de las universidades de Vigo y Santiago de Compostela, entre centros radicados en Murcia y entre la Universidad de Granada y la de Jaén. La consulta de la versión online del mapa permite obtener detalles bajo demanda. En el mapa quedan señalados los países extranjeros en los que se detectan trabajos en colaboración con centros españoles. Se les asigna colores y tamaños, con los mismos criterios comentados para los centros españoles. Se ha ubicado cada país en el centroide de las coordenadas geográficas de las instituciones que colaboran con las instituciones. El nodo representa los valores bibliométricos 
agregados de tamaño e impacto normalizado (NI) de las publicaciones del país correspondiente.

\section{Discusión y conclusión}

La producción española en ciencia de los alimentos agregada por centros de investigación e instituciones de educación superior, destaca por volumen, valores de impacto normalizado y tamaño de sus producciones de Exc10 y Exc10 RG. Es un sistema institucional que desde el punto de vista organizativo y con carácter general, está fragmentado y es heterogéneo, lo que puede dificultar la coordinación de la investigación en ciencia de los alimentos. Se detecta actividad en 660 instituciones con al menos un trabajo, de las que 110 son empresas con producciones inferiores a 22 trabajos. La presencia de universidades españolas en el sector de investigación de ciencia de los alimentos es destacada.

Basándonos en los datos Scopus, y en indicadores de percentiles, se han identificado aquellos centros que tienen una actividad sostenida en la categoría temática y un rendimiento superior al esperado. En ocasiones son centros que no tienen una elevada producción, por lo que con este enfoque se normaliza por el tamaño de las producciones. Este método, ya practicado en trabajos similares, posibilita la identificación tanto de los centros con rendimiento superior al esperado, como de aquellos otros que no alcanzan ese nivel.

El mapa elaborado, ubica los lugares de producción de las investigaciones en el espacio geográfico y dota de apariencia visible al flujo de conocimientos que se detecta por las actividades de colaboración interinstitucional. Desde este punto de vista, el mapa ofrece más detalles y precisa de forma más exacta, comparado con otros enfoques, la información bibliométrica derivada del análisis de actividades de producción, impacto de citación y redes de coautoría en la categoría temática. Como los indicadores que se pueden representar en un mapa son elección de sus autores, este método permite también ubicar centros españoles que publican trabajos altamente citados. El mapa presentado en este artículo no es sino un ejemplo, entre las varias posibilidades de dimensiones candidatas a poder ser representadas en un mismo dominio temático.

La información que contiene revela la importancia que tiene la proximidad en los procesos de aprendizaje y creación de conocimiento. Proximidad entendida en el sentido que le otorga Boschma (2005): cognitiva, organizativa, social, institucional y geográfica. Así, se pueden reconocer en el mapa, los vínculos de colaboración existentes entre dos instituciones relacionadas por unos intereses temáticos comunes, como con el ejemplo entre el Instituto de Ciencias de la Vid y el Vino (ICVV) en Logroño, y el Instituto de la Vid y el Vino en Castilla la Mancha (Ivicam), en Tomelloso, Ciudad Real.

También se reconocen aspectos derivados de estructuras organizativas de investigación creadas ex profeso, como los centros catalanes integrados al amparo del Programa de Centros de Investigación de Cataluña (Cerca). Otros aspectos como los geográficos o institucionales, se aprecian con claridad en el mapa y ya se han comentado en los resultados.
El mapa esboza también las diferencias interterritoriales, las asimetrías y diferencias regionales correspondientes al desarrollo del Estado de las autonomías. Mayor actividad de investigación en ciencia de los alimentos en comunidades como por ejemplo Madrid, Cataluña, Valencia o Baleares donde menor peso tiene la inversión pública sobre PIB, y menor actividad en aquellas Comunidades donde el peso de las inversiones públicas sobre PIB son mayores, como por ejemplo Extremadura, ambas Castillas, Asturias, Aragón y Andalucía. Estas últimas son precisamente las comunidades autónomas donde se concentra el mayor porcentaje de la renta agraria en España.

El método elegido presenta limitaciones y sólo puede comprenderse como un método aproximado de medición de la influencia intelectual en el área. No existe un consenso sobre cómo delimitar las publicaciones que representen adecuadamente la misma área de investigación. Existen modos diversos de contabilizar las publicaciones, y con procedimientos diferentes de contabilidad se obtienen resultados distintos en los valores institucionales. Puede haberse deslizado algún error de visualización en la clasificación de las direcciones a partir de las contenidas en las direcciones de los trabajos o en la asignación de las coordenadas. Sin embargo, sin esos indicadores bibliométricos no es posible construir esta cartografía.

\section{Agradecimientos}

Este trabajo ha sido financiado por el Plan Nacional de Investigación Científica, Desarrollo e Innovación Tecnológica 2012-2015 y el Fondo Europeo de Desarrollo Regional (Feder), como parte del proyecto de investigación CSO2013-40530-R, y por la Junta de Extremadura y la Consejería de Educación Ciencia y Tecnología y el Fondo Social Europeo como parte de la beca para Grupos de Investigación GR10019.

\section{Bibliografía}

Alfaraz, Pablo H.; Calviño, Amalia-Mirta (2004). "Bibliometric study on food science and technology: Scientific production in iberian-american countries (1991-2000)". Scientometrics, v. 61, n. 1, pp. 89-102.

http://dx.doi.org/10.1023/B:SCIE.0000037365.53469.91

Bornmann, Lutz; De-Moya-Anegón, Félix; Leydesdorff, Loet (2012). "The new excellence indicator in the World Report of the SCImago Institutions Rankings 2011". Journal of informetrics, v. 6, n. 2, pp. 333-335.

http://www.lutz-bornmann.de/icons/Exclndi.pdf

http://dx.doi.org/10.1016/j.joi.2011.11.006

Bornmann, Lutz; Leydesdorff, Loet (2011). "Which cities produce more excellent papers that can be expected? A new mapping approach, using Google Maps based on statistical significance testing". Journal of the American Society for Information Science and Technology, v. 62, n. 10, pp.1954-1962.

http://arxiv.org/ftp/arxiv/papers/1103/1103.3216.pdf http://dx.doi.org/10.1002/asi.21611

Borsi, Balázs; Schubert, András (2011). “Agrifood research in Europe: a global perspective". Scientometrics, v. 86, n. 1, pp. 133-154. 
http://dx.doi.org/10.1007/s11192-010-0235-3

Boschma, Ron (2005). "Proximity and innovation: a critical assessment". Regional studies, v. 39, n. 1, pp. 61-74. http://dx.doi.org/10.1080/0034340052000320887

Cañas-Guerrero, Ignacio; Mazarrón, Fernando R.; PouMerina, Ana; Calleja-Perucho, Cruz; Díaz-Rubio, Gonzalo (2013). "Bibliometric analysis of research activity in the "Agronomy" category from the Web of Science, 1997-2011". European journal of agronomy, v. 50, October, pp. 19-28. http://dx.doi.org/10.1016/j.eja.2013.05.002

Chinchilla-Rodríguez, Zaida; Olmeda-Gómez, Carlos (2010). "Producción y colaboración científica en agroalimentación". En: Sanz-Menéndez, Luis; Cruz-Castro, Laura. Análisis sobre ciencia e innovación en España: Anuario Icono. Madrid: Fundación Española para la Ciencia y la Tecnología, pp. 366-399. ISBN: 9788469362938

http://icono.fecyt.es/informesypublicaciones/Documents/ Analisis_Ciencia_Innovacion.pdf

De-Moya-Anegón, Félix; Guerrero-Bote, Vicente P.; Bornmann, Lutz; Moed, Henk (2013). "The research guarantors of scientific papers and the output counting: A promising new approach". Scientometrics, v. 97, n. 2, pp. 421-434.

http://www.lutz-bornmann.de/icons/guarantor.pdf http://dx.doi.org/10.1007/s11192-013-1046-0

Frenken, Koen; Hardeman, Sjoerd; Hoekman, Jarno (2009). "Spatial scientometrics: towards a cumulative research program". Journal of informetrics, v. 3, n. 3, pp. 222-232. http://dx.doi.org/10.1016/j.joi.2009.03.005
Guerrero-Bote, Vicente P.; De-Moya-Anegón, Félix (2015). "Analysis of scientific production in food science from 2003 to 2013". Journal of food science.

http://dx.doi.org/10.1111/1750-3841.13108

Levy-Mangin, Jean-Pierre; Varela-Mallou, Jesús (2003). Análisis multivariable para las ciencias sociales. Madrid: Prentice Hall. ISBN: 9788420537276

Muñoz-Cidad, Cándido; Sosvilla-Rivero, Simón (2012). FIAB. Informe económico 2012. Madrid: Federación Española de Industrias de la Alimentación y Bebidas. FIAB. ISBN: 9788469535080

http://www.fiab.es/archivos/documentoMenu/ documentomenu_20130510124729.pdf

Olmeda-Gómez, Carlos (2014). "Visualización de información". El profesional de la información, mayo-junio, v. 23, n. 3, pp. 213-219.

http://dx.doi.org/10.3145/epi.2014.may.01

Rehn, Catharina; Wadskog, Daniel; Gornitzki, Carl; Larsson, Agnes (2014). Bibliometric indicators - Definitions and usage at Karolinska Institutet. Karolinska Institutet.

http://kib.ki.se/sites/default/files/bildarkiv/Dokument/ bibliometric_indicators_2014.pdf

Yegros-Yegros, Alfredo (2011). Contribución del Programa Nacional de Tecnología de Alimentos al desarrollo de la actividad científica en Ciencia y Tecnología de Alimentos en España. Tesis Doctoral. Universidad Politécnica de Valencia. http://www.ingenio.upv.es/sites/default/files/tesis/ TE49_1_Tesis-Alfredo.pdf

\section{Inforảrea}

Ayudamos a tu organización en la transformación digital y el gobierno de la información

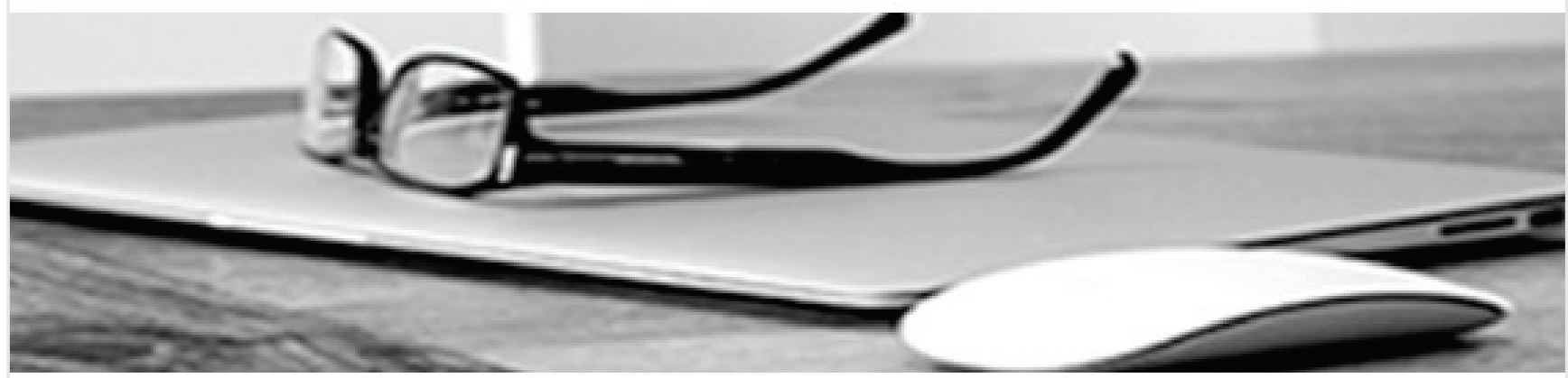

* Consultoría estratégica en gestión y gobierno de la información

" Gestión documental y "records management"

* Gestión de contenidos, intranets corporativas y entornos de colaboración

* Estudios especializados

Clientes satisfechos, cientos de empresas nacionales e internacionales y más de 30 años de experiencia son la mejor garantía de nuestra reputación. 